\title{
La aportación de IMS Learning Design a la creación de recursos pedagógicos reutilizables
}

\author{
The contribution of IMS Learning Design to the creation of reusable \\ learning resources
}

\author{
David Griffiths \\ University of Bolton. Bolton. UK \\ d.e.griffiths@bolton.ac.uk \\ Josep Blat \\ Universitat Pompeu Fabra, Barcelona, España \\ josep.blat@upf.edu \\ Rocío García \\ Universitat Pompeu Fabra, Barcelona, España \\ rocio.garcia@upf.edu \\ Sergio Sayago \\ Universitat Pompeu Fabra, Barcelona, España \\ sergio.sayago@upf.edu
}

\begin{abstract}
Resumen
En este artículo se discute el concepto y la utilidad del objeto de aprendizaje, proponiendo que el factor clave es su uso, y no tanto sus características. Después, se revisan las actuales especificaciones de eLearning para la definición de estos objetos, indicando sus ventajas y desventajas. La especificación IMS Learning Design (LD) es analizada con más detalle, destacando su contribución al debate de los objetos reutilizables de aprendizaje con referencia a experiencias en la Universitat Po mpeu Fabra. Finalmente, se señala la importancia y necesidad de herramientas apropiadas y se indican las iniciativas actuales que potencian la implementación y uso de la especificación IMS-LD.
\end{abstract}

Palabras clave: Objeto didáctico reutilizable, lenguaje de modelización educativo, IMS Learning Design, SCORM, pedagogía, herramientas.

\begin{abstract}
The concept of learning object is discussed, and its value assessed. It is proposed that the key factor is use, rather than the inherent characteristics of the object. The eLearning specifications which can be used to define reusable learning objects are then reviewed, and their advantages and disadvantages outlined. IMS Learning Design (LD) is discussed in greater detail, together with its origins in the earlier OUNL EML specification, and analyzed in terms of the debate surrounding learning objects. The feasibility of using IMS LD to support reusability is examined, with reference to studies carried out at Universitat Pompeu Fabra. Appropriate tooling is identified as a key issue, and current initiatives for supporting implementation and adoption of the specification are indicated.
\end{abstract}

Keywords: Reusable learning object, educational modeling language, IMS Learning Design, SCORM, pedagogy, tools. 


\section{Los contenidos reutilizables y los objetos didácticos}

La reutilización es un hecho cotidiano en la educación presencial. A nivel personal, los profesores reutilizan apuntes y esquemas didácticos. A nivel institucional, los libros, y otros recursos docentes, se emplean en una amplia variedad de instituciones educativas, con alumnos y entornos pedagógicos muy distintos, tal como indica Downes (2002). Sin embargo, cuando se trata de la educación a distancia, se presenta una problemática importante a dos niveles. A nivel práctico, la reutilización es difícil de conseguir; a nivel teórico, no hay una terminología clara y aceptada. Un aspecto que contribuye considerablemente a estas dificultades es el debate abierto en torno al análisis del proceso del aprendizaje presencial. Como mencionamos en una publicación anterior (Griffiths \& Garcia, 2003), hay una contradicción entre dos conceptos del aprendizaje. Por un lado, la manera informal con la que se habla de la transmisión de información y conocimientos en los procesos comunicativos y educativos; por otro, el consenso en la tradición constructivista, en la cual se sitúan los presentes autores, que plantea que la comprensión y los conceptos se construyen en la mente del que aprende.

En el primer caso, cuando se habla de la comunicación, el discurso emplea frecuentemente la metáfora del conducto, descrita por Lakoff y Johnson:

\section{LAS IDEAS (o SIGNIFICADOS) SON OBJETOS}

\section{LAS EXPRESIONES LINGÜÍSTICAS SON CONTENEDORES}

\section{LA COMUNICACION IMPLICA ENVIAR ${ }^{1}$}

En el mundo de la educación esta metáfora se ha incorporado en la redacción de planes de estudios que tienen "contenidos" que se tienen que "impartir" a los estudiantes. En cambio, se sabe que a nivel biológico una idea no es un objeto (Maturana \& Varela, 1980), y que tampoco se puede enviar. En otro ámbito intelectual, la tradición del constructivismo, que tiene sus orígenes en la obra de Vygotsky, indica que las ideas se construyen en el cerebro del que aprende (por ejemplo Vygotsky (1978), y autores más recientes como Bruner (1983) o, aún más claramente, von Glaserfeld (1996)). Es decir, que dentro de un sistema educativo nominalmente constructivista, como es, por ejemplo, el sistema escolar público español regido por la LOGSE, sabemos que los estudiantes construyen sus propios conocimientos, pero hablamos y actuamos como si fueran los profesores quienes les entregan los conocimientos.

En la educación presencial las intervenciones de los profesores pueden resolver esta contradicción. Un sinfín de intervenciones didácticas, muchas de las cuales son espontáneas y no documentadas, ayudan a los estudiantes a construir su propia comprensión. Así se mantiene la ficción de un discurso que (adaptando un poco la metáfora de Lakoff) representa el conocimiento como un objeto, contenido en recursos, que se puede entregar al estudiante. Son estas intervenciones didácticas las que permiten que un libro se pueda reutilizar durante varios cursos para enseñar una materia a miles de escolares en muchos centros. En eLearning, en cambio, estas intervenciones suelen desaparecer. La infraestructura para la relación profesor-alumno es generalmente pobre, o, incluso, inexistente.

\footnotetext{
${ }^{1}$ En el ingles original: IDEAS (or MEANINGS) ARE OBJECTS; LINGUISTIC EXPRESSIONS ARE CONTAINERS; COMMUNICATION IS SENDING.
}

La aportación de IMS Learning Design a la creación de recursos pedagógicos reutilizables. 
Dada esta falta de claridad (de si los recursos en la educación presencial contienen conocimientos o no), no es ninguna sorpresa que el concepto de recurso didáctico en eLearning también sea motivo de múltiples interpretaciones. Ejemplificando una de las aproximaciones, Wylie plantea "This is the fundamental idea behind learning objects: instructional designers can build small (relative to the size of an entire course) instructional components that can be reused a number of times in different learning contexts. Additionally, learning objects are generally understood to be digital entities deliverable over the Internet" (Wiley, 2000). En esta definición Wiley sostiene que un objeto didáctico reutilizable es un componente que tiene un contenido de instrucción. Siendo conscientes que simplificamos posturas pedagógicas sutiles, se puede decir que Wiley resuelve la contradicción afirmando que el conocimiento sí reside realmente en el recurso, y que el diseñador educativo lo ha puesto allí. Pero esto no debería ser aceptable por un constructivista con aspiraciones de coherencia. Por otro lado, el IEEE sostiene que "a learning object is defined as any entity, digital or non-digital, that may be used for learning, education or training" (IEEE, 2002). Esta definición evita situar el conocimiento en el objeto, perdiendo todo el poder discriminante. Es decir, según esta definición, cualquier objeto físico, texto, o recurso informático, se podría utilizar para fines educativos y sería un objeto didáctico.

Este panorama tan divergente de discursos sobre la educación, que son muy difíciles de cambiar, nos obliga a plantearnos si el concepto de objeto didáctico es realmente útil. Pensamos que este concepto es tan difícil de definir porque es una pieza clave en una visión de la educación fundamentalmente ambigua, y el eLearning es donde la contradicción entre dos conceptos de la adquisición del conocimiento se ha hecho más patente y dañina por la falta de la presencia moderadora del profesor presencial. Desde este punto de vista, creemos que el siguiente esquema sobre los objetos pedagógicos sería más fácil de manejar:

1. hay recursos de todo tipo, digitales y físicos, que se pueden emplear en la educación

2. hay recursos diseñados específicamente para la educación (por ejemplo, los manuales) así como se pueden utilizar en educación los de otros ámbitos (textos literarios, muestras químicas, fotos, etc.).

3. los recursos tienen un carácter educativo en virtud de su uso por alumnos en actividades educativas, no por sus cualidades internas.

El punto 3 quizás no deja muy claro cómo se podría incluir la reutilización de los objetos didácticos en este esquema; aparte del nivel banal y obvio que un recurso se puede utilizar muchas veces en cualquier contexto. Planteamos este tema en una sección posterior, pero primero pasamos a examinar la relación entre las especificaciones principales de eLearning y la reutilización de recursos educativos.

\section{SCORM y sus implicaciones pedagógicas}

En la sección anterior hemos expuesto cómo las ideas sobre la reutilización de recursos pedagógicos están condicionadas por concepciones más generales sobre educación. En esta sección examinamos cómo las especificaciones de eLearning, que pueden facilitar la interoperabilidad y por tanto la reutilización, también condicionan la naturaleza de esta reutilización.

La aportación de IMS Learning Design a la creación de recursos pedagógicos reutilizables. David Griffiths, Josep Blat, Rocio Garcia y Sergio Sayago

Página 3 de 11 
Las primeras especificaciones trataban de apoyar la localización de recursos de aprendizaje con el esquema de metadatos desarrollado por el proyecto Ariadne. Este esquema se incorporó posteriormente a IMS-LOM (Learning Object Metadata). Esta especificación permite, principalmente, describir recursos y facilitar su localización. IMS $^{2}$ Global Learning Inc, que incluye muchas empresas y universidades importantes, es la organización que ha tenido más éxito en los últimos años en establecer especificaciones de eLearning que sean ampliamente aceptadas por la industria y por el mundo académico. Utilizando otra especificación de IMS, Content Packaging, se puede asegurar que los recursos llegan íntegros a puerto, sin perder ningún elemento asociado, y con un manifiesto que especifica el contenido que ha sido empaquetado. Los metadatos incluyen una serie de conceptos con valor educativo, tales como la edad de los alumnos, dificultad de aprendizaje, y el papel del usuario, entre otros. Sin embargo, no permiten definir ninguna actividad a desarrollar con los recursos. Esto es el objetivo de IMS Simple Sequencing. IMS Simple Sequencing permite la definición de recorridos pedagógicos que el usuario puede seguir para atravesar los recursos, con bifurcaciones según unas condiciones simples. El usuario también puede contestar preguntas definidas siguiendo la especificación IMS Question and Test Interoperability, bien como recurso de aprendizaje o de evaluación.

Estas especificaciones configuran la base de lo que se puede definir como actividades pedagógicas en el conocido perfil de aplicación SCORM (Shareable Courseware Object Reference Model). SCORM es el estándar de eLearning con mayor penetración en el mercado educacional. Se tiene que admitir que la funcionalidad descrita es muy pobre si se compara con la riqueza de una experiencia educativa presencial, pero también es preciso destacar que el proceso de redacción de especificaciones es lento, y aún no se ha completado. Ya que el aprendizaje es un campo complejo, es natural que los desarrolladores de especificaciones hayan empezado por las funcionalidades más básicas y fáciles de implementar. Estas funcionalidades, además, son generalmente aceptadas como necesarias en cualquier proceso educativo, ya que los pedagogos de todos los colores están de acuerdo en que es útil poder entregar recursos, programarlos, y administrar cuestionarios.

Otro tema más controvertido es si, además de ser necesaria, esta funcionalidad es también suficiente para un eLearning satisfactorio, y en este sentido podemos citar los persuasivos argumentos de (Friesen, 2004), donde se sostiene que no es ni mucho menos suficiente, ya que se limita al caso de un estudiante trabajando solo ante un ordenador, sin aprovechar las capacidades comunicativas de la red. No hay ningún soporte para la colaboración entre estudiantes, ni para el diálogo con el profesor, y las actividades se limitan a lectura de recursos en varias secuencias, y cuestionarios. Dadas estas limitaciones, la siguiente afirmación de ADL, el organismo responsable de SCORM, provoca cierta reflexión "The SCORM Content Aggregation Model represents a pedagogically neutral means for designers and implementers of instruction to aggregate learning resources for se afirma que SCORM es pedagógicamente neutro, no sólo respecto a la entrega de conocimientos, sino incluso respecto a la experiencia del aprendizaje. Parece, pues, que SCORM resuelve la contradicción de concepciones de la adquisición del conocimiento decantándose definitivamente por la metáfora del conducto. Mientras que un pedagogo que quiera implementar recursos educativos

\footnotetext{
${ }^{2}$ Para más información sobre IMS, consulten www.ims -project.org . IMS originalmente significaba Instructional Management Systems, pero ahora oficialmente las siglas carecen de significado.
}

La aportación de IMS Learning Design a la creación de recursos pedagógicos reutilizables. 
reutilizables con una pedagogía más sofisticada, empleando quizás elementos de colaboración y comunicación, no encuentra allí el soporte adecuado.

\section{La contribución de IMS-LD}

Hasta ahora hemos indicado dos problemas que influyen negativamente en la reutilización de los recursos educativos: la confusión sobre el concepto de objeto didáctico y el soporte limitado a la pedagogía ofrecido en las especificaciones de eLearning. Ahora pasamos a considerar la contribución de una especificación más reciente, IMS Learning Design, que puede resolver estas dificultades. Para entender el papel de esta especificación es útil tener una comprensión básica de sus orígenes.

En 1997 la Open University of the Netherlands (OUNL) decidió convertir todos sus cursos en cursos on-line. Los cursos existentes empleaban una variedad de enfoques pedagógicos. La Universidad los clasificó y empezó a implementar unas plantillas representativas para intentar dar soporte a todas estas categorías pedagógicas. Rápidamente se constató que todos los profesores tenían su propia visión pedagógica, y que necesitaban casi tantas plantillas como profesores. Por otro lado, aunque había muchas descripciones pedagógicas de los cursos, en la práctica todas consistían en combinaciones de tres elementos básicos: recursos educativos, múltiples personas actuando en varios roles, y actividades pedagógicas. El EML (Educational Modelling Language), introducido por la OUNL, permite definir estos tres elementos y así especificar la estructura de una Unit of Learning, UoL, mediante un documento XML. Para que alumnos y profesores utilicen una UoL se tiene que crear una instancia con unas personas concretas, y mediante una aplicación player se coordina la publicación de los materiales y actividades a las personas precisas en el instante oportuno.

IMS, consciente de las limitaciones pedagógicas de las especificaciones existentes, empezó el proceso de desarrollo de una especificación para la definición de aspectos pedagógicos, pero ya que EML existía y funcionaba decidieron adaptarlo en lugar de crear una especificación totalmente nueva.

El resultado es una nueva especificación, IMS Learning Design. Aunque presenta cambios importantes de estructura y enfoque, sus conceptos básicos y capacidades son muy similares a los de EML. En este artículo no tenemos espacio para explicar los detalles de la especificación, y el lector interesado puede encontrar más información sobre IMS Learning Design en www.unfold-project.net. Para nuestra argumentación actual, los aspectos importantes de IMS Learning Design son los siguientes:

- ofrece soporte para múltiples alumnos, y contempla la comunicación entre ellos

- representa el papel de profesor

- permite combinar recursos educativos con actividades pedagógicas, y con las interacciones entre personas en diferentes roles.

Estas capacidades facilitan que el diseñador de las UoL pueda definir, por ejemplo, actividades de aprendizaje basado en problem-based Learning (enseñanza basada en problemas), como las del prototipo EML que diseñamos en el proyecto SCOPE (2003).

La aportación de IMS Learning Design a la creación de recursos pedagógicos reutilizables. 
Por otro lado, un ejemplo en el IMS Learning Design Best Practice Guide ${ }^{3}$, Versailles Negotiation, nos muestra como IMS Learning Design permite modelar y coordinar una actividad de negociación con varios pasos de actividades en grupo y la documentación adecuada. Una vez que se ha creado la estructura de la actividad de negociación, ésta se puede adaptar a un tema totalmente distinto, con otros recursos y participantes, pero manteniendo la estructura de la actividad, lo que permite la reutilización de las actividades pedagógicas (más allá de los contenidos).

Antes hemos formulado que los recursos adquieren carácter educativo cuando unos alumnos los emplean para aprender. Ahora afirmamos que IMS Learning Design permite que podamos convertir este uso en un objeto reutilizable. Ya no se considera la existencia de conocimientos en el recurso que se pueden transmitir a un estudiante receptor, sino que los recursos son un elemento esencial, pero no suficiente, en un proceso más amplio y complejo de aprendizaje. El recurso solamente puede ser usado para aprendizaje cuando se combina con actividades realizadas por personas en roles, es decir (en términos de IMS Learning Design), cuando forma parte de una Unit of Learning. La UoL puede tener muchas formas distintas, incluso con el mismo recurso. Por ejemplo, un texto que describa una ciudad puede formar parte de una Unit of Learning (UoL) tratando de historia, o de literatura, o de lengua, o de formación de educadores, entre otros temas, y cada contexto tendría actividades y / o roles distintos. Las diferentes UoLs serían reutilizables por profesores distintos, con cohortes distintas de alumnos.

Como se puede observar, una UoL es un elemento mucho más complejo que un objeto de SCORM, y puede ofrecer oportunidades de aprendizaje mucho más ricas. Reiteramos que, desde nuestra perspectiva, no tiene sentido hablar de un objeto didáctico reutilizable (con énfasis en didáctico) en ausencia de actividades pedagógicas de personas en roles. Por tanto, la definición de IEEE "a learning object is defined as any entity, digital or non-digital, that may be used for learning, education or training", sería insuficiente (aunque si se substituye objeto didáctico por recurso educativo sería acertada). Una UoL diseñada con IMS Learning Design sí que podría considerarse como un objeto didáctico reutilizable, pero dada la confusión en relación a este término, quizás es más aconsejable abandonarlo.

Las implicaciones de este cambio en la manera de entender los objetos didácticos no son simplemente didácticas. Los bancos de recursos didácticos tradicionales no sólo incluyen documentos, sino que también incluyen esquemas didácticos, que indican cómo el profesorado ha empleado los recursos con sus estudiantes para conseguir diferentes resultados pedagógicos. Muchas veces esto es lo que más interesa a los profesores, y no el recurso en sí. La pregunta clave no es "¿qué materiales didácticos puedo encontrar para enseñar esta materia?" sino "¿qué actividades han realizado mis compañeros para enseñar esta materia?". Utilizando IMS Learning Design se puede representar este tipo de información sobre el uso en el aula, que combina recursos con actividades y roles, cosa que no es posible utilizando SCORM.

\footnotetext{
${ }^{3}$ Disponible a http://www.imsglobal.org/learningdesign/index.cfm
}

La aportación de IMS Learning Design a la creación de recursos pedagógicos reutilizables. David Griffiths, Josep Blat, Rocio Garcia y Sergio Sayago 


\section{La factibilidad de utilizar IMS Learning Design para definir recursos didácticos reutilizables}

IMS Learning Design tiene el potencial de facilitar nuevas funcionalidades didácticas para un eLearning basado en estándares y especificaciones. Hasta cierto punto, se puede afirmar también que es una solución factible, ya que OUNL imparte desde hace unos años cursos utilizando EML y la aplicación player Edubox, con resultados positivos (Tattersall, 2004). En este contexto institucional de una universidad a distancia prestigiosa, se puede invertir una cantidad importante de dinero en el mantenimiento de un equipo de soporte técnico para la creación de UoLs reutilizables. No hay ningún motivo para pensar que la situación sería distinta para IMS Learning Design, y de hecho la relevante empresa Blackboard ha llegado a un acuerdo con OUNL para adaptar Edubox de forma que funcione con IMS Learning Design (IMS Global Learning, 2003).

Sin embargo, es cierto que hay muchas instituciones que no pueden permitirse tal soporte técnico. En este caso la creación de UoLs puede ser más difícil de asumir. En la Universitat Pompeu Fabra (UPF) se han realizado unas pruebas iniciales para examinar la factibilidad de utilizar esta tecnología para definir recursos didácticos reutilizables. En primer lugar se desarrollaron dos UoLs en el marco del proyecto SCOPE (Griffiths et al., At the press) para crear el prototipo de un curso de educación continuada para especialistas médicos en gastroenterología y hepatología. Utilizando artículos procedentes de la revista G\&H Continuada (G\&H Continuada, 2004) como recursos, se crearon dos itinerarios, uno de aprendizaje basado en problemas, y otro de pedagogía tradicional tipo "leer y evaluar la comprensión". El prototipo, definido en EML y ejecutado en Edubox, funcionó muy bien, pero el coste de su creación fue muy elevado.

Las UoLs se pueden crear utilizando editores estándares de XML, desde básicos hasta avanzados, como sería XML Spy, o herramientas específicas. En nuestro caso, utilizamos LD Editor de Perot ${ }^{4}$. LD Editor es una herramienta diseñada para técnicos, que requiere un conocimiento bastante profundo de la especificación LD para crear UoLs. En el momento de la creación de estas UoLs no existía ninguna herramienta adecuada para especialistas en la materia docente sin conocimiento en LD pudieran crear UoLs directamente. Por otro lado, adaptar el diseño cuando se utilizaba el player EduBox ${ }^{5}$ era más difícil que cuando se utilizaba HTML (aunque EduBox funcionaba bien para ejecutar las UoLs). Este es un factor importante cuando se quiere mantener un estilo propio (como es el caso de la editorial de $G \& H$ Continuada).

También se desarrolló una UoL de apoyo online para una parte de un curso presencial de diseño de interfaces en los estudios de Informática de la UPF. En este caso el coste de elaboración era prohibitivo comparado con la facilidad y bajo coste de implementación del mismo conjunto de actividades en el contexto presencial.

EML se desarrolló inicialmente en un contexto determinado. Una universidad a distancia necesitaba trabajar con una gama amplia de enfoques pedagógicos, y con múltiples alumnos, sólo con la posibilidad de interactuar con el profesorado de manera no presencial. No existía una especificación de eLearning adecuada, y por tanto, era

\footnotetext{
${ }^{4}$ Agradecemos la generosa colaboración de Perot Netherlands poniendo a nuestra disposición una versión beta de esta herramienta, y también EduBox player para correr las UoLs.

${ }^{5}$ Vea (Koper \& Tattersall, 2005)
}

La aportación de IMS Learning Design a la creación de recursos pedagógicos reutilizables. 
preciso desarrollarla. En un contexto institucional con necesidades y capacidades similares, el uso de IMS Learning Design es una opción atractiva, ya que la única alternativa es la creación de otro sistema igualmente costoso diseñado expresamente, y sin perspectivas de interoperabilidad con otros sistemas. En cambio, en las experiencias piloto de la UPF, el uso de la especificación era deseable, pero no esencial y seguramente no ofrecía ventajas añadidas sustanciales relativas a su coste. La capacidad de representar una variedad de enfoques pedagógicos de apoyo a clases presenciales, o la interacción de múltiples alumnos, son prestaciones atractivas, pero no son esenciales ya que se pueden sustituir por alternativas no tecnológicas en la actividad docente presencial, simplemente con ayuda de recursos impresos o páginas HTML. A ello se puede añadir el factor de la escasa renovación pedagógica de la universidad. En el caso del prototipo del curso a distancia, el modelo pedagógico era bastante simple, de un alumno aislado y dos enfoques pedagógicos, para ello pueden ser suficientes dos plantillas - aunque una variedad mayor de pedagogías, y unas herramientas más sencillas de usar pueden hacer atractiva la opción de utilizar plenamente IMS LD. En estas condiciones el coste de implementar sobre la base de IMS LD era prohibitivo porque sus prestaciones eran deseables, pero no esenciales y las herramientas muy primitivas y complejas. Creemos que consideraciones de este tipo pueden ser también válidas para contextos semejantes, y un resultado es que el número actual de UoLs que se han creado es limitado. Como consecuencia hay una fuerte limitación en la práctica al potencial que tiene IMS LD para facilitar el intercambio de recursos reutilizables que realmente tienen un componente didáctico.

\section{La importancia de la mejora de las herramientas}

Señalemos que algunas de las limitaciones descritas anteriormente para aplicar IMS-LD pueden ser transitorias, ya que se deben principalmente al diseño de las herramientas disponibles actualmente, y no tanto a la naturaleza de la especificación. Por ejemplo, actualmente es frecuente que los autores de UOLs visualicen el XML, pero con las nuevas herramientas que empiezan a salir es previsible que no necesiten saber de la existencia de ficheros XML. La producción de UoLs con las herramientas existentes es similar a la producción de páginas en los primeros años del Web, cuando personas con suficientes conocimientos técnicos creaban los sitios con un editor de texto directamente en HTML (y/o Javascript o Java). La producción de sitios y páginas web solamente se ha generalizado a partir de varias generaciones de editores más sofisticados, con cada vez más soporte conceptual y mecanismos que aíslan a los usuarios de la necesidad de aprender los elementos y la estructura de HTML (y más aún Javascript o Java). Seguramente un proceso similar será necesario para que existan herramientas de IMSLD accesibles a profesores y pedagogos, y los mismos alumnos, pero en el caso de IMS-LD es previsible que sea más difícil por dos razones. En primer lugar, la especificación de IMS-LD es mucho más compleja (que HTML), pero además IMS-LD tiene que interaccionar con otras especificaciones. Por ejemplo un profesor quizás necesitará incorporar una evaluación en una actividad pedagógica. La evaluación se tendrá que definir con IMS-QTI, que es la especificación relacionada con evaluación, y no con IMS-LD, pero no es razonable que el profesor sepa nada de esta distinción. Por lo tanto será preciso desarrollar herramientas orientadas a tareas, y no únicamente orientadas hacia especificaciones de eLearning, por muy útiles que sean aplicaciones de este tipo.

La aportación de IMS Learning Design a la creación de recursos pedagógicos reutilizables. 


\section{El estado del desarrollo de herramientas}

Teniendo en cuenta que IMS Learning Design se publicó al principio de 2003, no es sorprendente que no exista aún un conjunto de herramientas maduro ${ }^{6}$. En esta sección indicamos algunos aspectos del soporte para la creación de herramientas que auguran un buen futuro. Para una visión más general, en (Griffiths et al., 2005) se presenta una tipología, y una revisión de las herramientas que están disponibles o en fase de desarrollo.

En 2002 se fundó el Valkenburg Group, uno de cuyos objetivos es coordinar la producción de herramientas para IMS-LD. El grupo definió la Valkenburg Architecture $^{7}$. Esta arquitectura proporciona una estructura general que guía el desarrollo de las herramientas. En 2004 varios miembros del Valkenburg Group han conseguido financiación de la Comisión Europea para UNFOLD, un proyecto concebido para dar apoyo a la adopción de IMS-LD. Este apoyo se articula mediante dos acciones principales: la compilación y eventual creación de recursos para incrementar la conciencia de la especificación entre los posibles usuarios, y la organización de Comunidades de Práctica (Communities of Practice). Los individuos implicados en IMS-LD muchas veces trabajan de manera aislada, sin interactuar con colegas que se interesan en los mismos temas. Por ello no consiguen tanta información como sería conveniente, e incluso necesario; se pierden oportunidades de colaboración, y hay una tendencia a la duplicación de trabajo, lo que se intenta remediar con las Comunidades de Práctica. En UNFOLD, las Comunidades de Práctica son tres: Desarrolladores de Sistemas, Diseñadores de UoLs, y Profesores y Proveedores de Educación. Todas tienen actividades online, y también reuniones, seminarios y talleres técnicos presenciales. También hay flujos de información entre las tres comunidades, ya que los resultados de unas forman la infraestructura de otras. Finalmente hay una conexión estrecha con los desarrolladores de la especificación. Este tipo de colaboración se puede potenciar con proyectos de desarrollo FOSS (Free and Open Source Software). Hay varios proyectos importantes de este tipo relacionados con IMS-LD, como el RELOAD Editor, y el CopperCore Learning Design Engine, un motor Open Source para crear players de IMSLD. Estas y similares iniciativas permiten cierto optimismo sobre el futuro de la utilización más general de IMS-LD. Cuando maduren, confiamos en que existirán las condiciones adecuadas para la creación fácil de recursos reutilizables que sean realmente didácticos para la educación.

Fecha de cierre de la redacción del artículo: 23 de junio de 2005

Griffiths, D., Blat, J., Garcia, R., y Sayago, S. (2016). La aportación de IMS Learning Design a la creación de recursos pedagógicos reutilizables. RED. Revista de Educación a Distancia, 50. Consultado el (dd/mm/aaaa) en http://www.um.es/ead/red/50

\footnotetext{
${ }^{6}$ Para una revisión amplia de las herramientas disponibles se puede consultar Griffiths et al (Griffiths, Blat, Garcia, Vogten, \& Kwong, 2005). El Web de UNFOLD, www.unfold-project.net, también irá dando información actualizada sobre las herramientas disponibles.

${ }^{7}$ La Valkenburg Reference Architecture está disponible en el Web de UNFOLD, a http://www.unfold project.net:8085/UNFOLD/general_resources_folder/tools/architecture
}

La aportación de IMS Learning Design a la creación de recursos pedagógicos reutilizables. David Griffiths, Josep Blat, Rocio Garcia y Sergio Sayago

Página 9 de 11 


\section{Referencias}

ADL (2003). SCORM. Version 1.3 Application Profile - Working Draft.

Bruner, J. (1983). Actual Minds, Possible Worlds. Harvard University Press.

Downes, S. (2002). Learning Objects. Recuperado de: http://www.downes.ca/files/Learning_Objects.htm [2004, 5th August].

Friesen, N. (2004). Three Objections to Learning Objects. In R. McGreal (Ed.), Online Education Using Learning Objects. London: Routledge/Falmer.

G\&H Continuada. DOYMA. Recuperado de: http://www.ghcontinuada.com/ [2004, 8th September].

Griffiths, D., Blat, J., Garcia, R., Sayago, S., Casado, F., y Martínez, J. (In press). Challenges in the wider adoption of EML/LD: two exploratory case studies. En R. Koper \& C. Tattersall (Eds.), Learning Design: modelling and implementing network-based education \& training. Springer Verlag.

Griffiths, D., Blat, J., Garcia, R., Vogten, H., y Kwong, K. (2005). Learning Design Tools. En R. Koper \& C. Tattersall (Eds.), Learning Design: modelling and implementing network-based education \& training. (pp. 109-135). Springer Verlag.

Griffiths, D., \& Garcia, R. (2003). Commentary on Rob Koper, Combining re-Usable Learning Resources to Pedagogical Purposeful Units of Learning, Chapter 5 of: Reusing Online Resources: A Sustainable Approach to eLearning, (Ed.) Allison Littlejohn. Journal of Interactive Media in Education, Special Issue on Reusing Online Resources, 1.

IEEE. (2002). Draft Standard for Learning Object Metadata, Sponsored by the Learning Technology Standards Committee of the IEEE. New York.

IMS Global Learning. (2003). Learning Design Specification Version 1. Recuperado de: http://www.imsglobal.org/learningdesign/ [2005, 21st June].

Koper, R., \& Tattersall, C. (Eds.). (2005). Learning Design: modelling and implementing network-based education \& training. Springer.

Maturana, H., \& Varela, V. (1980). Autopoiesis and Cognition: The Realization of the Living (Vol. 42): Dordecht: D. Reidel Publishing Co. SCOPE. (2003). Project Showcase. Recuperado de: http://www.tecn.upf.es/scope/showcase/ [1905, 21st June 2005].

Tattersall, C. (2004). Setting up a Learning Design system in your institution. OUNL experiences: UNFOLD Project.

Von Glaserfeld, E. (1996). Radical Constructivism. Routledge Falmer.

La aportación de IMS Learning Design a la creación de recursos pedagógicos reutilizables.

David Griffiths, Josep Blat, Rocio Garcia y Sergio Sayago

Página 10 de 11 
Vygotsky, L. (1978). Mind in Society: The Development of Higher Psychological Processes. Cambridge, MA: Harvard University Press.

Wiley, D. A. E. (2000). Connecting learning objects to instructional design theory: A definition, a metaphor, and a taxonomy. The Instructional Use of Learning Objects: Online Version. Association for Instructional Technology. Recuperado de: http://reusability.org/read/chapters/wiley.doc [2004, 5th August]. 